\title{
BEYOND 2D FOR BRAIN-COMPUTER INTERFACES: TWO 3D EXTENSIONS OF THE P300-SPELLER
}

\author{
Saman Noorzadeh, Bertrand Rivet and Christian Jutten \\ GIPSA-lab, CNRS UMR-5216, \\ Grenoble University, Grenoble, France
}

\begin{abstract}
This paper, investigates the use of a 3D setting for BrainComputer Interface (BCI) by implementing the 3D interface for the P300-Speller device. The 3D configurations were implemented using two different approaches which are called Natural $3 D$ and Parallel 2D. The theoretical analysis concerning these two approaches are provided considering the modifications in speed, accuracy, and capacity. The experimental results on subjects who tested the 3D interfaces are then provided to validate the theoretical analysis.
\end{abstract}

Index Terms - 3D BCI, P300-Speller Interface, Virtual Keyboard, 3D Stereoscopic Images

\section{INTRODUCTION}

BCI has been commonly the most suitable application to allow communication, or control of external devices only based on brain brain activities [1,2]. These activities are recorded non-invasively as the EEG signal [3]. P300-Speller is a BCI device which works based on Event-Related Potentials (ERPs) appearing in EEG signal, and has been first developed by Farwell and Donchin [4] with an interface containing a matrix of symbols, and sequential random flashes on rows and columns as depicted in Figure 1.

If a user keeps a mental count of the number of the stimulus flashes of a specific symbol, the intensifications of the row and the column containing that symbol elicits a positive wave about $300 \mathrm{~ms}$ after the stimulus in his/her EEG signal, which is called P300 evoked potentials.

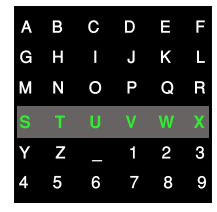

(a) Flash on the $4^{\text {th }}$ row

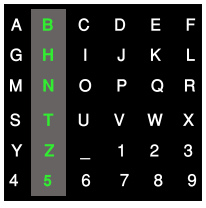

(b) Flash on the $2^{\text {nd }}$ column
Figure 1: The classical 2D interface; the flashes of (a) and (b) indicate the letter ' $T$ '.

Most of the researches on the P300-speller have focused on enhancing the signals by removing the artifacts $[5,6,7]$, or on classification of P300 from non-P300 evoked potentials [8]. More recently, the flashing paradigm on rows and columns has been modified, for example motion of rows and columns is used as the stimulus instead of flashes [9]. Similarly, others used variations on motion, colors or flash patterns $[10,11,12]$. Another paradigm, called checkerboard paradigm, is proposed in [13] in which the standard matrix of symbols is virtually superimposed on a checkerboard to avoid the wrong detection of a character and its adjacent one. For almost the same reason, [14] has also proposed a new flashing paradigm, which will be used in our 3D virtual keyboards.

We consider here the use of a 3D virtual keyboard instead of the classical 2D one. Firstly, we intend to investigate the application of 3D settings in BCI devices by verifying its performance on the P300-Speller. This can also increase the user's acceptability of the device, which is a factor that has not been taken into much consider. Secondly, considering the virtual keyboard in 3 dimensions causes changes in flashing paradigm and can increase the device's speed and capacity.

This paper is organized as follow: The methods that are used to implement a 3D virtual keyboard are presented in Section 2. In Section 3 the theoretical analysis of the proposed 3D extensions are presented. The mentioned theories are then evaluated according to the results obtained through the experiments in Section 4. Finally, Section 5 sums up the techniques which were presented in previous sections.

\section{3D EXTENSIONS OF P300 BASED BCI}

Here, we consider modifying the interface of the P300-Speller by expanding the $2 \mathrm{D}$ keyboard to 3 dimensions. Two approaches of flashing strategies are implemented and compared in this paper: "natural 3D"(section 2.1) and "parallel 2D" (section 2.2).

\subsection{Natural 3D}

The natural generalization of the classical 2D interface is to reorganize the symbols in a three dimensional matrix. This is implemented according to 3D stereoscopic methods [15, 16, $17,18]$ in 3 depth layers, and plane flashes are used as stimuli. This configuration is reffered to as natural $3 D$ in which each 


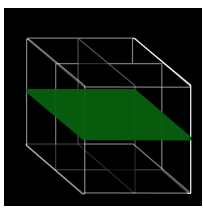

(a) Flash on a row

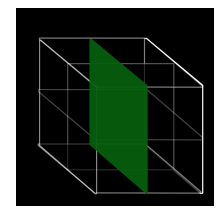

(b) Flash on a column(c) Flash on a depth
Figure 2: Flashes corresponding to the planes of Natural 3D interface with 3 rows, 3 columns, and 3 depths.

symbol is the intersection of a row, a column, and a depth plane (Figure 2).

\subsection{Parallel 2D}

Another flashing approach is considered by treating the different depths of the 3D keyboard as separate 2D keyboards. Using such a strategy (denoted as parallel $2 D$ ), the flashes on each layer are the classical row and column flashes. The depth is coded by a delay between the stimuli onsets on the different layers. Figure 3 illustrates this statement with two layers by showing the stimuli timelines: the dotted line corresponds to one of the depth levels and the continuous line corresponds to the other one. As one can see the interlacing flashes lead to independent or parallel layers.

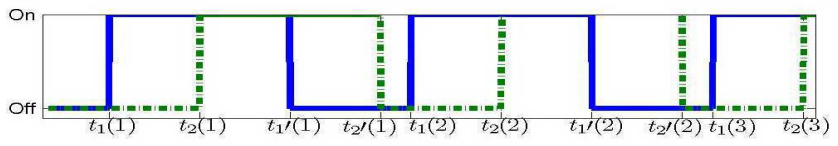

Figure 3: Parallel flashes on 2 depth levels in Parallel 2D interface.

It has to be considered that the number of 2D keyboards that can function in parallel is limited, first because of the limited frame rate of the imaging device and secondly, the delay between layers should not be too small for ensuring a good detection. In this way we can avoid the wrong detection of depth to a large amount. The flashing paradigm of [14] is also implemented on the parallel 2D keyboard to avoid the wrong detections between adjacent symbols.

\section{THEORETICAL ANALYSIS AND COMPARISON OF 3D INTERFACES}

In this section, theoretical analysis of the proposed 3D interfaces is provided by comparing their accuracy (Section 3.1), their speed (Section 3.2) and their capacity (Section 3.3).

\subsection{Accuracy}

Let's consider the accuracy of the classical 2D interface as a reference. To detect the symbol that the user intends to spell, a permutation of flashes on all rows and columns (so that each row and column is flashed once and only once) is needed and is referred to as repetition. Usually one repetition is not enough for an acceptable accuracy; therefor, there are several repetitions with different permutations. Now, let $p(R=r, C=c)$ denotes the probability that the row $r$ and the column $c$ are jointly detected. Considering the classical 2D interface, the probability of detecting the row can be assumed to be independent from that of the column:

$$
p(R=r, C=c)=p(R=r) p(C=c) .
$$

As a consequence, assuming that the detection probability of a correct row and a column is equal and is denoted $P \in[0,1]$, the accuracy of the classical 2D interface is expressed as

$$
A c c_{2 D}=p\left(R=r_{t}, C=c_{t}\right)=P^{2},
$$

where $r_{t}$ (resp. $c_{t}$ ) is the true row (resp. column) containing the selected symbol. In natural 3D the probability of detecting the symbol in row plane $r$, column plane $c$, and depth plane $d$, considering the same assumptions of equation (1), is given by:

$$
\text { Acc }_{\text {natural } 3 D}=p\left(R=r_{t}, C=c_{t}, D=d_{t}\right)=P^{3} .
$$

In parallel 2D, assuming that the depth is coded by the time delay between layers, the probability of detecting a symbol as the intersection of row $r$ and column $c$ in the layer $d$ is independent of $d$. So the accuracy is:

Acc $_{\text {parallel } 2 D}=p\left(R=r_{t}, C=c_{t}, D=d_{t}\right)=P^{2}$.

According to equations 2, 3, and 4 , the detection rates of classical and parallel 2D were estimated to be equal, and it is higher than the estimated accuracy of natural 3D.

\subsection{Number of Flashes: Speed of the Interface}

Considering $S$ as the total number of symbols on the interface, a minimum of $N_{2 D}$ flashes are required in each repetition, to cover all rows and columns in classical 2D: $N_{2 D}=\left\lceil 2 \times S^{\frac{1}{2}}\right\rceil$ where $\lceil\cdot\rceil$ is the ceiling function. For the proposed row-column-depth stimulation of natural 3D interface this number would be $N_{\text {natural } 3 D}=\left\lceil 3 \times S^{\frac{1}{3}}\right\rceil$ On each layer of the parallel row-column stimulation (parallel 2D) with d layers, the minimum number of flashes would be: $N_{\text {parallel2D }}=\left\lceil 2 \times\left(\frac{S}{d}\right)^{\frac{1}{2}}\right\rceil$

Figure 4 compares the minimum number of flashes. As one can see, with more than nine symbols into the interface $(S=9)$, the classical 2D interface needs more flashes than the natural 3D; and for less than 85 symbols, the parallel 2D interface needs more flashes than the natural 3D. On the contrary, the parallel 2D always requires less flashes than the classical 2D one. 


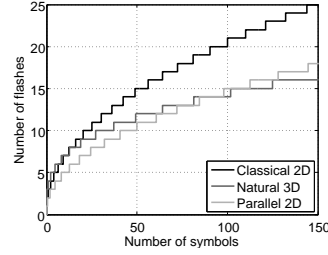

(a) Number of Flashes

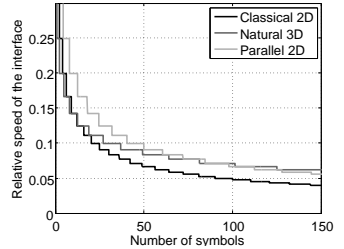

(c) Relative Speed
Figure 4: Comparison of the interfaces. Figure 4.a: relative speed $F$ of the interfaces defined as $N \times T$ where $T$ is the ISI.

\section{3. capacity}

A more relevant performance measure to quantize the information transfer rate (ITR) [19] is the capacity, which is defined as equation (5) for a $\mathrm{S}$-symbol interface.

$C=\log _{2}(S)+A c c \log _{2}(A c c)+(1-A c c) \log _{2}\left(\frac{1-A c c}{S-1}\right)$

Where $A c c$ is the accuracy of the interface. The capacity $C$ is expressed in bits per repetition. Due to the different number of flashes, the duration of a repetition in each approach (classical 2D, natural 3D or parallel 2D) is different from the other. So the comparison is more relevant considering the bit rate $B$, in bits per second, defined as

$$
B=C \times F
$$

or the relative bit rate $B^{\prime}$, expressed in bits per flash (because of a same duration for every flash), defined as

$$
B^{\prime}=\frac{C}{N}
$$

As shown in Figure 5, the bit rates of the natural 3D and parallel 2D interfaces are always larger than the bit rate of the classical 2D interface for a given accuracy. For parallel $2 \mathrm{D}$, this is due to the smaller number of flashes with the same accuracy. This improvement factor is given by $N_{2 D} / N_{\text {parallel } 2 D}$. However, since the relationships between the accuracy and the marginal probability of good detection are nonlinear, the bit rate of the natural 3D interface is only larger than the bit rate of the classical 2D interface for large marginal probability $P$. This threshold decreases as the number of symbols increases.

\section{RESULTS AND ANALYSIS}

The preliminary experiments with almost a small number of subjects just intend to prove the functionality of 3D settings in BCIs and validate the theoretical results that have been explained. Two comparisons are presented: the classification accuracy and the capacity of the two 3D extensions.

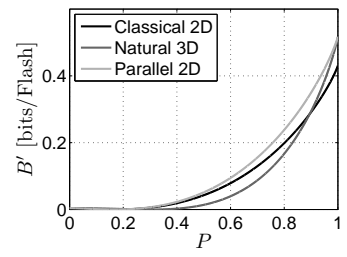

(a) 36 symbols

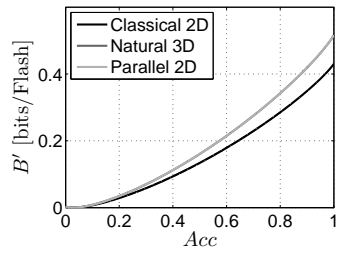

(c) 36 Symbols

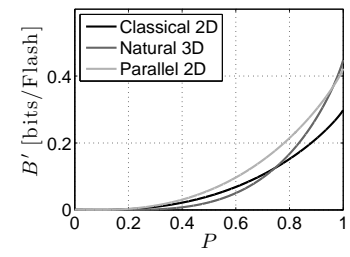

(b)144 Symbols

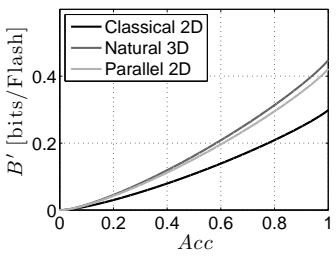

(c) 144 Symbols
Figure 5: Relative bit rate per flash $B^{\prime}$ of equation (7) against marginal probability of good detection $P$ (Figures 5.a, 5.b) and against the accuracy (Figures 5.c, 5.d). In Figure 5.d, $B_{\text {natural } 3 D}^{\prime}=$ $B_{\text {parallel } 2 D}^{\prime}$ since $N_{\text {natural } 3 D}=N_{\text {parallel2D. }}$.

\subsection{Data, Feature Extraction, and Classification}

The data is collected from 16 volunteers between 22 and 34 years old, with normal stereoscopic vision and neurological state. 8 participants have been subjected to the natural 3D interface, and the other 8 ones to the Parallel 2D. They all have done an experiment on classical 2D before 3D, so that the results can be compared. For each approach there is a train and a test session. The interfaces are implemented using OpenGL, in 5 repetitions with ISI equal to 133 milliseconds, and a duty cycle of $50 \%$ for the flashes. The interfaces characteristics are summarized in table 1. The EEG is recorded

\begin{tabular}{|c|c|c|}
\hline Interface & \# Symbols & row $\times$ column $\times$ depth \\
\hline Classical 2D & 36 & $2 \times 2$ \\
\hline Natural 3D & 27 & $3 \times 3 \times 3$ \\
\hline Parallel 2D & 32 & $4 \times 4 \times 2$ \\
\hline
\end{tabular}

Table 1: Implemented Interfaces

via 16 active electrodes with g.USBamp device from g.tec. The signal is sampled at a rate of $1200 \mathrm{~Hz}$, and filtered by a bandpass filter in the frequency band between 1 and $12 \mathrm{~Hz}$ with a fourth order Butterworth filter. To enhance the signals, the spatial filters are estimated [20, 21, 7] from the training data. The temporally and spatially filtered signal is then categorized into two target and non-target epochs, and is used as the two-class data to train the classifier: Bayesian Linear Discriminant Analysis (BLDA) [22]. K-fold cross-validation is adopted: the database is divided into 40 symbols used to the training and 10 symbols for the tests. This partition is per- 
formed randomly 2000 times for each testing configuration.

\subsection{Accuracy and Marginal Probability}

The classification accuracies of the two 3D extensions are compared in Figure 6. First of all, one notices that the classification accuracy obviously increases with the number of repetitions. Then, as expected in the theoretical section (Section 3.1), it is worth noting that the classification accuracy of the classical 2D is larger than the classification of the natural 3D extension, while they are the same between the classical 2D and the parallel 2D interfaces. It should be noted that the results concerning classical 2D in different figures are the same in average. And the small difference is due to the fact that they are tested on different subjects.

To verify the theoretical assumptions on the marginal probabilities, P, Figure 7 is depicted. Figure 7.a proves the equal marginal probability hypothesis for row and column of the natural 3D and they are similar to the marginal probability of good detection with the classical 2D interface; however, this is not true for marginal probability of good detection of depth, which is smaller than that of row

Regarding Figure 7.b, for parallel 2D the two assumptions regarding the marginal probabilities are well validated: first, these probabilities are similar on rows and columns, and also similar to that of classical 2D. Second, the marginal probability of detecting the depth is equal to one after at least 3 repetitions. This proves that the depth is well coded by the flashing paradigm.

\subsection{Capacity}

Figure 8 compares the capacity of 3D approaches with the classical 2D. For a better understanding, the figures show directly the difference of new interfaces' capacities with the classical 2D's. It is depicted that the capacity of the parallel 2D interface is higher than that of classical 2D which is confirmed by the theoretical analysis (Figure 5). The variance of this result can be interpreted as the visual effect while looking at 3D images [23].

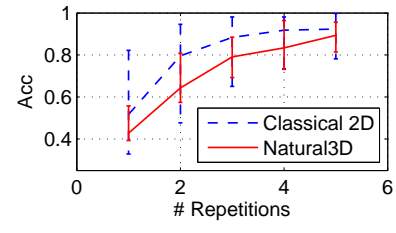

(a) Natural 3D

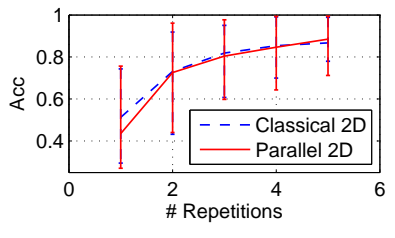

(c) Parallel 2D
Figure 6: Accuracy against the number of repetitions. The median accuracies are plotted, and the error bars extend from the $10 \%$ quantile to the $90 \%$ quantile.

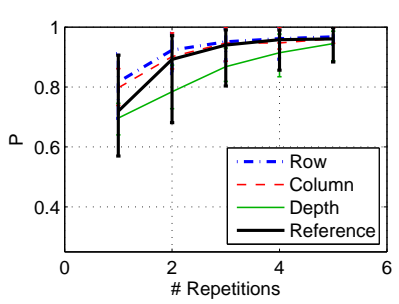

(a) Natural 3D

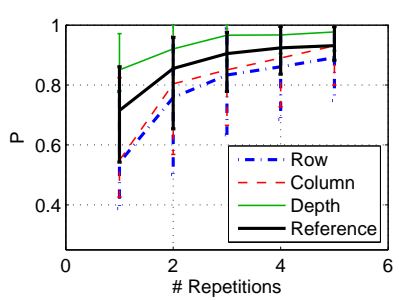

(c) Parallel 2D
Figure 7: Marginal probability $(P)$ against the number of repetitions.

However, Figure 8.a shows that the classical 2D has higher capacity comparing the natural 3D. As it is already proved that the natural 3D has less accuracy comparing classical 2D (Figure 6.a), it is natural that the capacity does not follow Figure 6.d which assumes the same accuracy for all approaches.

\section{CONCLUSION}

The paper presented two 3D extensions of the classical 2D interface represented in [4]. The methods are called Natural $3 D$ and Parallel $2 D$. Experiments on 16 subjects showed that the proposed interfaces have several advantages over the classical 2D interface. One of these advantages is the ergonomics of the device. according to a survey from the subjects, we found that $75 \%$ of the subjects preferred 3D keyboards to $2 \mathrm{D}$ because of its comfort and higher ergonomic features.

The next advantage is the speed which is proven to be higher in both proposed interfaces comparing classical 2D, since they need smaller number of flashes.

As the modification of the P300-Speller is done just on the interface part, we do not expect any accuracy improvement in the theoretical part, and also this is confirmed in the experiments. This accordance of theoretical results with the experimental ones is missing only for the marginal probability of detecting the depth which was not equal to detection of

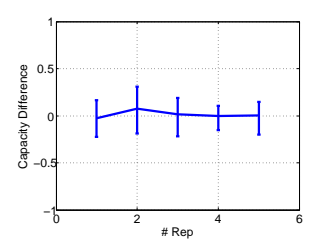

(a) Natural 3D

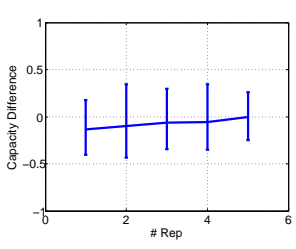

(c) Parallel 2D
Figure 8: Comparison of relative bitrates. $B_{M a x}^{\prime}$ is the maximum bitrate assuming a perfect accuracy (i.e. $A c c=100 \%$ ). 
other dimensions in natural 3D.

Finally, with the analysis of the capacity of the new interfaces, parallel 2D approach showed a better performance than the other ones.

The validation of theoretical results along with the increase of speed and accuracy mostly in the parallel 2D interface, can prove the functionality of the 3D settings not only in the p300-Speller device, but also in other BCI technologies which require more ergonomic features, as in virtual worlds [24].

\section{REFERENCES}

[1] L. R. Hochberg, M. D. Serruya, G. M. Friehs, J. A. Mukand, M. Saleh, A. H. Caplan, A. Branner, D. Chen, R. D. Penn, and J. P. Donoghue, "Neuronal ensemble control of prosthetic devices by a human with tetraplegia," Nature, vol. 442, pp. 164-171, 2006.

[2] P. R. Kennedy and R. A. Bakay, "Restoration of neural output from a paralyzed patient by a direct brain connection," Neuroreport, vol. 9, pp. 1707-1711, 1998.

[3] J. R. Wolpaw, N. Birbaumer, D. J. McFarland, G. Pfurtscheller, and T. M. Vaughan, "Brain-computer interfaces for communication and control," Clin. Neurophysiol, vol. 113, pp. 767791, 2002.

[4] L. A. Farwell and E. Donchin, "Talking off the top of your head: toward a mental prosthesis utilizing event-related brain potentials," Electroencephalography and Clinical Neurophysiology, vol. 70, no. 6, pp. 510-523, December 1988.

[5] H. Serby, E. Yom-Tov, and G.F. Inbar, “An improved P300-based braincomputer interface," IEEE Transactions on Neural Systems and Rehabilitation Engineering, vol. 13, no. 1, pp. 89-98, March 2005.

[6] Matthias Kaper, Peter Meinicke, Ulf Grossekathoefer, Thomas Lingner, and Helge Ritter, "BCI Competition 2003-Data Set IIb: Support Vector Machines for the P300 Speller Paradigm," IEEE Trans Biomed Eng., vol. 51, no. 6, pp. 1073-1076, June 2004.

[7] B. Rivet and A. Souloumiac, "Optimal linear spatial filters for evoked potentials: an asymptotic performance analysis," Signal Processing, vol. 93, pp. 387-398, 2013.

[8] Dean J Krusienski, Eric W Sellers, François Cabestaing, Sabri Bayoudh, Dennis J McFarland, Theresa M Vaughan, and Jonathan R Wolpaw, "A comparison of classification techniques for the P300 Speller," Journal of Neural Engineering, vol. 3, no. 4, pp. 299-305, December 2006.

[9] S M M Martens, N J Hill, J Farquhar, and B Scholkopf, "Overlap and refractory effects in a brain-computer interface speller based on the visual p300 event-related potential," Journal of Neural Engineering, vol. 6, no. 2, pp. 026003 (9pp), 2009.

[10] B. Hong, F. Guo, T. Liu, X. Gao, and S. Gao, "N200-speller using motion-onset visual response," Clin Neurophysiol., vol. 120, pp. 16581666, 2009.

[11] K. Takano, T. Komatsu, N. Hata, Y. Nakajima, , and K. Kansaku, "Visual stimuli for the p300 brain-computer interface: A comparison of white/gray and green/blue flicker matrices," Clin Neurophysiol, 2009.

[12] Salvaris M. and Sepulveda F., "Visual modifications on the p300 speller bci paradigm," J Neural Eng., vol. 6, pp. 046011, 2009.

[13] G. Townsend, B.K. LaPallo, C.B. Boulay, D.J. Krusienski, G.E. Frye, C.K. Hauser, N.E. Schwartz, T.M. Vaughan, J.R. Wolpaw, and E.W. Sellers, "A novel p300-based brain-computer interface stimulus presentation paradigm: Moving beyond rows and columns," Clin Neurophysiol, vol. 121, no. 7, pp. 1109-1120, 2010.

[14] Hubert Cecotti and Bertrand Rivet, "One step beyond rows and columns flashes in the p300 speller: a theoretical description," Inter. Journal of bioelectromagnetism, vol. 13, no. 1, pp. 39-41, 2010.
[15] I. P. Howard and B. J. Rogers, Binocular Vision and Stereopsis, New York: Oxford Univ. Press, 1995.

[16] C. Wang and A. A. Sawchukg, "Disparity manipulation for stereo images and video," in Proceedings of Stereoscopic Displays and Applications XIX, 2008.

[17] T. M. Lambooij, W. A. IJsselsteijn, and I. Heynderickx, "Visual discomfort in stereoscopic displays: a review," in Stereoscopic Displays and Virtual Reality Systems XIV, 2007.

[18] R. Vlad., "State-of-the-art report on stereoscopic 3D issues with focus on quality," 2011, Deliverable MOOV3D project.

[19] J Kronegg, S Voloshynovskiy, and T. Pun, "Analysis of bit-rate definitions for braincomputer interfaces," in Proceedings of the 2005 inter. conference on human-computer interaction, 2005.

[20] B. Rivet, A. Souloumiac, G. Gibert, and V. Attina, "P300 speller braincomputer interface: Enhancement of P300 evoked potential by spatial filters," in EUSIPCO, 2008.

[21] B. Rivet, A. Souloumiac, V. Attina, and G. Gibert, "xDAWN algorithm to enhance evoked potentials: application to brain-computer interface," IEEE Trans Biomed Eng., vol. 56, 2009.

[22] U. Hoffmann, JM. Vesin, T. Ebrahimi, and K. Diserens, "An efficient P300-based brain-computer interface for disabled subjects," J Neurosci Methods, vol. 167, pp. 15-25, 2008.

[23] T. Shibata, T. Kawai, K. Ohta, M. Otsuki, N. Miyake, Y. Yoshihara, and T. Iwasaki, "Stereoscopic 3-D display with optical correction for the reduction of the discrepancy between accommodation and convergence," Journal of the Society for Information Display, vol. 13, pp. 665671, 2005.

[24] J.D. Bayliss, "Use of the evoked potential P3 component for control in a virtual apartment," IEEE Trans. of Neural Sys. and Rehab. Eng., vol. 11, pp. 113-116, 2008. 